\title{
Immature Squamous Metaplasia of Esophageal Glands Associated with Squamous Cell Carcinoma: Case Report
}

\section{Laurine Verset}

Institut Jules Bordet

Vincent Huberty

Hopital Erasme

Vincent Bourgeois

Centre Hospitalier Universitaire de Charleroi - Hopital Andre Vesale

Arnaud Lemmers

Hopital Erasme

Pieter Demetter ( $\nabla$ pieter.demetter@bordet.be )

Institut Jules Bordet

\section{Case Report}

Keywords: Endoscopic submucosal dissection, immature squamous metaplasia, esophagus, case report

Posted Date: July 16th, 2020

DOI: https://doi.org/10.21203/rs.3.rs-42268/v1

License: (9) This work is licensed under a Creative Commons Attribution 4.0 International License. Read Full License 


\section{Abstract}

Background: Esophageal immature squamous metaplasia is poorly reported in the literature. This entity can, however, be misinterpreted as high grade dysplasia or invasive squamous cell carcinoma and hence represent a potential pitfall.

Case presentation: Histopathological examination of a superficial esoophageal lesion removed by endoscopic submucosal dissection revealed a squamous cell carcinoma associated with immature squamous cell metaplasia arising from esophageal glands. Immunohistochemical stainings allowed to distinguish malignant from metaplastic cells.

Conclusions: Immunohistochemistry for Ber-EP4 is helpful in making the distinction between esophageal squamous cell carcinoma and immature squamous metaplasia. This can avoid overstaging and overtreatment, especially in early esophageal cancer.

\section{Background}

During the past decade, management of superficial esophageal squamous cell carcinoma (eSCC) using endoscopic submucosal dissection (ESD) and endoscopic mucosal resection (EMR) gained interest. The European Society of Gastrointestinal Endoscopy recommends ESD as the first option for superficial eSCC. EMR may be considered for lesions smaller than $10 \mathrm{~mm}$ if an en bloc resection can be assured [1].

Immature squamous metaplasia (ISM) is widely described in the cervix and represents epithelial changes from a single or multiple layers of reserve cells to an epithelium composed of three or more layers of cells with features of mature non keratinizing squamous epithelium [2]. This condition can be difficult to distinguish from high grade dysplasia, and immunohistochemistry is helpful in this distinction [3]. In the cervix, atypical immature metaplasia is considered as a morphological type of low grade squamous intraepithelial lesion in regard to immunoprofile as well as outcome [4].

Esophageal ISM is poorly described in literature but some authors made the hypothesis that esophageal ISM should be considered as a possible site of origin of eSCC development $[5,6]$.

Here we report the case of an ESD specimen with ESCC associated with ISM, discuss potential pitfalls and describe histopathological and immunohistochemical findings.

\section{Case Presentation}

A 58-year-old man was referred for management of a superficial esophageal lesion by ESD.

Macroscopic examination revealed a poorly circumscribed, erythematous, ulcerated, flattened lesion measuring $26 \mathrm{~mm}$. The ESD specimen was received fixed in 10\% formaldehyde and pinned out on a cork. The deep margin was inked in black and the lateral margins in green. The ESD specimen was completely embedded for microscopic analysis. 
Microscopic assessment revealed poorly differentiated eSCC admixed with extensive ISM (Fig. 1). ISM was found in the lamina propria and characterized by the presence of multilayered immature squamous cells covered by columnar and mucus-secreting epithelial cells. These cells formed islets with cystic appearance (Fig. 2a). ISM seemed to originate from ducts and displayed a ductal pattern reaching the superficial epithelium (Fig. 2a). Cells exhibited slight atypia with an increased nucleocytoplasmic ratio. Some mitoses were observed but they were limited to the basal area and no atypical mitosis were noted (Fig. 2b). Immature squamous cells were positive for p63 immunostaining while mucus-secreting epithelial cells were completely negative (Fig. 2c). PAS-diastase staining emphasized presence of mucus without goblet cells (Fig. 2d).

The presence of mucus-secreting cells suggests that ISM took its origin from ducts draining esophageal glands of the lamina propria. Ber-EP4 immunostaining revealed strong and membranous positivity (Fig. 2e). These islets were not invasive and collagen IV immunostaining confirmed the preserved basal membrane surrounding the islets (Fig. 2f). Ki-67 immunostaining showed proliferating cells limited to basal and parabasal compartments (Fig. $2 \mathrm{~g}$ ).

Submucosal glands were drained by ducts lined by monolayered cuboidal or stratified epithelium (Fig. 2h). Submucosal glands contained PAS-diastase positive cells while ducts did not (Fig. 2i). The ducts were positive for p63 immunostaining (Fig. 2j).

The poorly differentiated eSCC invaded the middle third of the submucosa. Tumoural cells were arranged into solid nests and showed a high level of atypia (Fig. 3a). Neoplastic cells were diffusely positive for p63 immunostaining (Fig. 3b) and Ki-67 demonstrated proliferating cells in the majority of the malignant cells (Fig. 3c). Collagen IV immunostaining showed a disrupted basal membrane (Fig. 3d). Neoplastic cells were negative for Ber-EP4 immunostaining (Fig. 3e). Deep and lateral margins were free. The eSCC was staged $\mathrm{pT} 1 \mathrm{~b}$ according to 8th TNM classification of the UICC.

\section{Discussion And Conclusions}

In this case a poorly differentiated eSCC was associated with florid ISM originating from esophageal gland ducts.

Two types of glands exist in the esophagus: esophageal cardiac-type glands located in the lamina propria which have a duct lined by gastric foveolar-like cells, and submucosal glands which are drained by ducts lined by a single layer of cuboidal epithelium in their proximal part but becoming stratified squamous when penetrating the muscularis mucosae and the epithelium before opening into the esophageal lumen [7]. In our case, stratification of ductal squamous epithelium of submucosal glands was already observed in the submucosa illustrating an early step of ISM. Furthermore, we illustrated that ISM displayed a ductal pattern. ISM with overlying mucus-secreting cells was only found in the lamina propria supporting the fact that this type of ISM developed from esophageal cardiac-type glands located in the lamina propria because the duct-lining cells may extend over the stratified squamous epithelium for variable distances [7]. 
Esophageal ISM is poorly reported in literature. About three decades ago, Takubo described reserve cell hyperplasia in $24 \%$ of 110 cases of which he analyzed histologically the complete mucosa of the esophagogastric junction [6]. Esophageal ISM could originate from stem cells that are present in esophageal glands and ducts; several studies support the notion that stem or progenitor cells within esophageal submucosal glands and/or ducts can generate both squamous and Barrett's columnar cells [8]. We observed diffuse positivity for Ber-EP4 immunostaining in ISM while tumoural cells as well as normal esophageal squamous epithelium were negative. In squamous epithelium of endodermal origin Ber-EP4 could not be demonstrated in mature squamous epithelium, but was focally present in immature, metaplastic and dysplastic squamous epithelium and occasionally also in hyperplastic squamous epithelium [9].

Esophageal ISM should not be confounded as intraductal spread of eSCC which can occur in nearly the quart of eSCC [10]. Intraductal spread of eSCC that extends to the submucosa should not be staged as submucosal infiltration and it has no impact on 5-year survival [11]. Similarly, esophageal ISM should not be considered as invasive cancer and we demonstrated in our case a preserved basal membrane surrounding the islets of ISM. Being aware of this pitfall can avoid overstaging and, so, overtreatment.

Classification of esophageal ISM as preneoplastic or dysplastic lesion (as in the cervix) is at this moment difficult due to lack of data in the literature.

In conclusion, extensive description of esophageal ISM is lacking in the literature; however, this entity needs to be recognized in order to avoid misinterpretation as cancer, and Ber-EP4 immunohistochemistry is a helpful immunohistochemical staining. Furthermore, data with regard to classification of esophageal ISM as possible preneoplastic or dysplastic entity are lacking. Co-occurence of ISM with eSCC as in the present case suggests that this subject merrits further investigation.

\section{Declarations}

Ethics approval/ Consent for publication : Patient signed a written informed consent upon admission to the hospital. Patients chose at admission whether to opt in or to opt out of providing consent, which affirms that physicians have the right to use patient's surplus biological material for research. Consent has been established by the local ethics committee (ethics committee Erasme-ULB) and is in accordance with Belgian and International law (Helsinki declaration).

Availability of data and materials: not applicable.

The authors declare that they have no competing interests.

Funding : not applicable

Authors' contributions: VH and AL performed ESD technique, LV realized macroscopic examination, LV and PD carried out microscopic examination. LV, VH, AL and PD wrote the manuscript. All authors read and approved the final manuscript. 


\section{References}

1. Pimentel-Nunes P, Dinis-Ribeiro M, Ponchon T, Repici A, Vieth M, De Ceglie A, et al. Endoscopic submucosal dissection: European Society of Gastrointestinal Endoscopy (ESGE) Guideline. Endoscopy. 2015;47:829-54. doi:10.1055/s-0034-1392882.

2. Vooijs GP, van Aspert-van Erp AJ, Bulten J. Benign Proliferative Reactions, Intraepithelial Neoplasia, and Invasive Cancer of the Uterine Cervix. In: Bibbo M, Wilbur DC, editors. Comprehensive Cytopathology. 3rd ed. Philadelphia: Elsevier; 2008. pp. 131-212.

3. Regauer S, Reich 0. CK17 and p16 Expression Patterns Distinguish (Atypical) Immature Squamous Metaplasia From High-Grade Cervical Intraepithelial Neoplasia (CIN III). Histopathology. 2007;50:629-35. doi:10.1111/j.1365-2559.2007.02652.x.

4. Duggan MA, Akbari M, Magliocco AM. Atypical Immature Cervical Metaplasia: Immunoprofiling and Longitudinal Outcome. Hum Pathol. 2006;37:1473-81. doi:10.1016/j.humpath.2006.05.013.

5. Takubo K, Tsuchiya S, Fukushi K, Shirota A, Mitomo Y. Dysplasia and Reserve Cell Hyperplasia-Like Change in Human Esophagus. Acta Pathol Jpn. 1981;31:999-1013. doi:10.1111/j.14401827.1981.tb02013.x.

6. Takubo K. Squamous Metaplasia With Reserve Cell Hyperplasia in the Esophagogastric Junction Zone. Acta Pathol Jpn. 1981;31:349-59. doi:10.1111/j.1440-1827.1981.tb01379.x.

7. El-Zimaity H, Riddell RH. Esophagus. In: Mills SE, editor. Histology for Pathologists. 4th ed. Philadelphia: Wolters Kluwer/Lippincott Williams\&Wilkins; 2012. pp. 614-20.

8. Zhang W, Wang DH. Origins of Metaplasia in Barrett's Esophagus: Is this an Esophageal Stem or Progenitor Cell Disease? Dig Dis Sci. 2018;63:2005-12. doi:10.1007/s10620-018-5069-5.

9. Rossen K, Thomsen HK. Ber-EP4 immunoreactivity depends on the germ layer origin and maturity of the squamous epithelium. Histopathology. 2001;39:386-89. doi:10.1046/j.1365-2559.2001.01235.x.

10. Takubo K, Takai A, Takayama S, Sasajima K, Yamashita K, Fujita K. Intraductal Spread of Esophageal Squamous Cell Carcinoma. Cancer 1987;59:1751-57. doi:10.1002/10970142(19870515)59:10<1751::aid-cncr2820591013>3.0.co;2-i.

11. Tajima Y, Nakanishi Y, Tachimori Y, Kato H, Watanabe H, Yamaguchi H, et al. Significance of Involvement by Squamous Cell Carcinoma of the Ducts of Esophageal Submucosal Glands. Analysis of 201 Surgically Resected Superficial Squamous Cell Carcinomas. Cancer 2000;89:248-54. doi:10.1002/1097-0142(20000715)89:2<248::aid-cncr7>3.0.co;2-q.

\section{Figures}




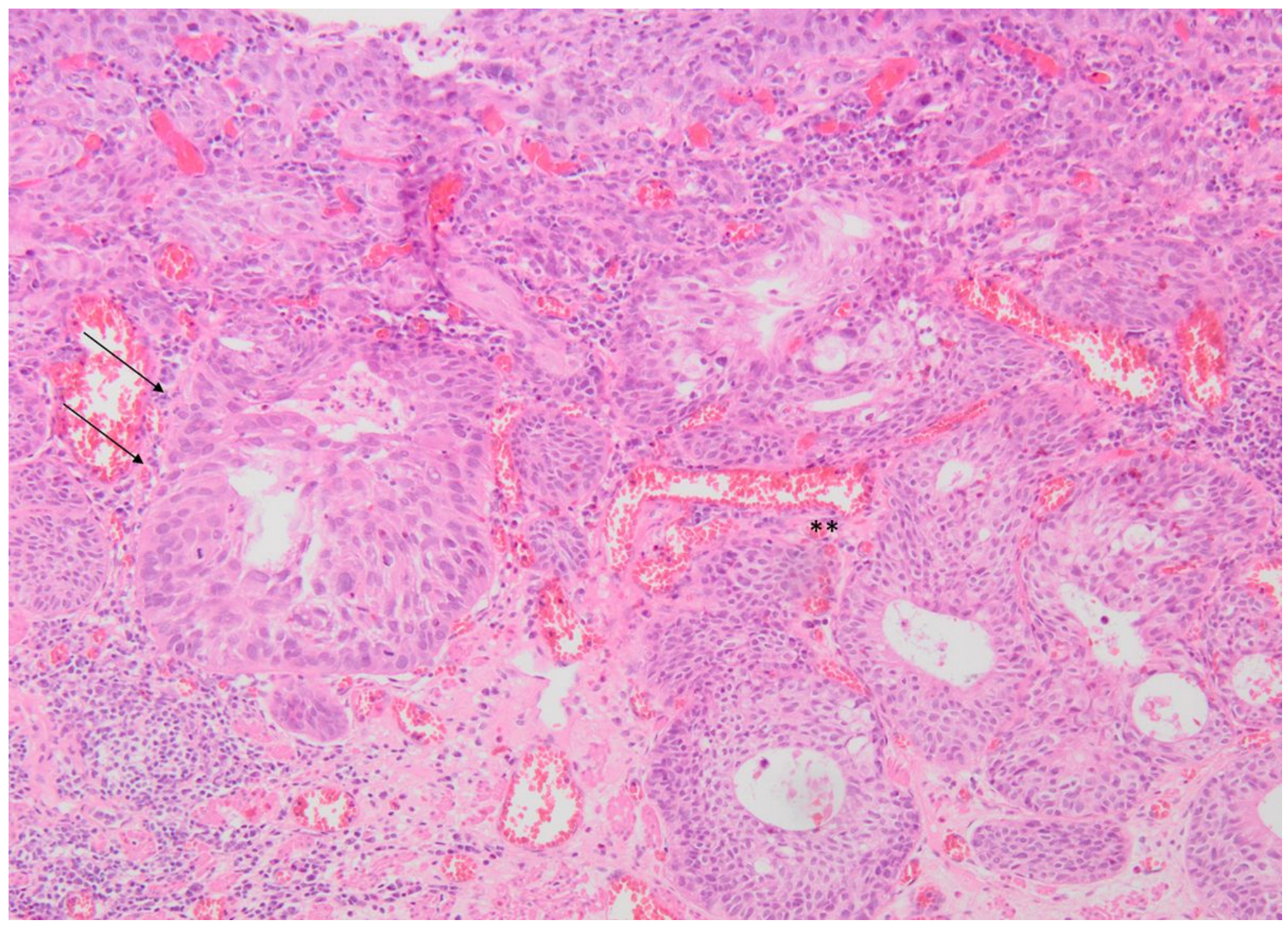

Figure 1

ISM (asterisk) intermingled with eSCC (arrows) (100x). Note the difference with regard to atypia between the two entities. 

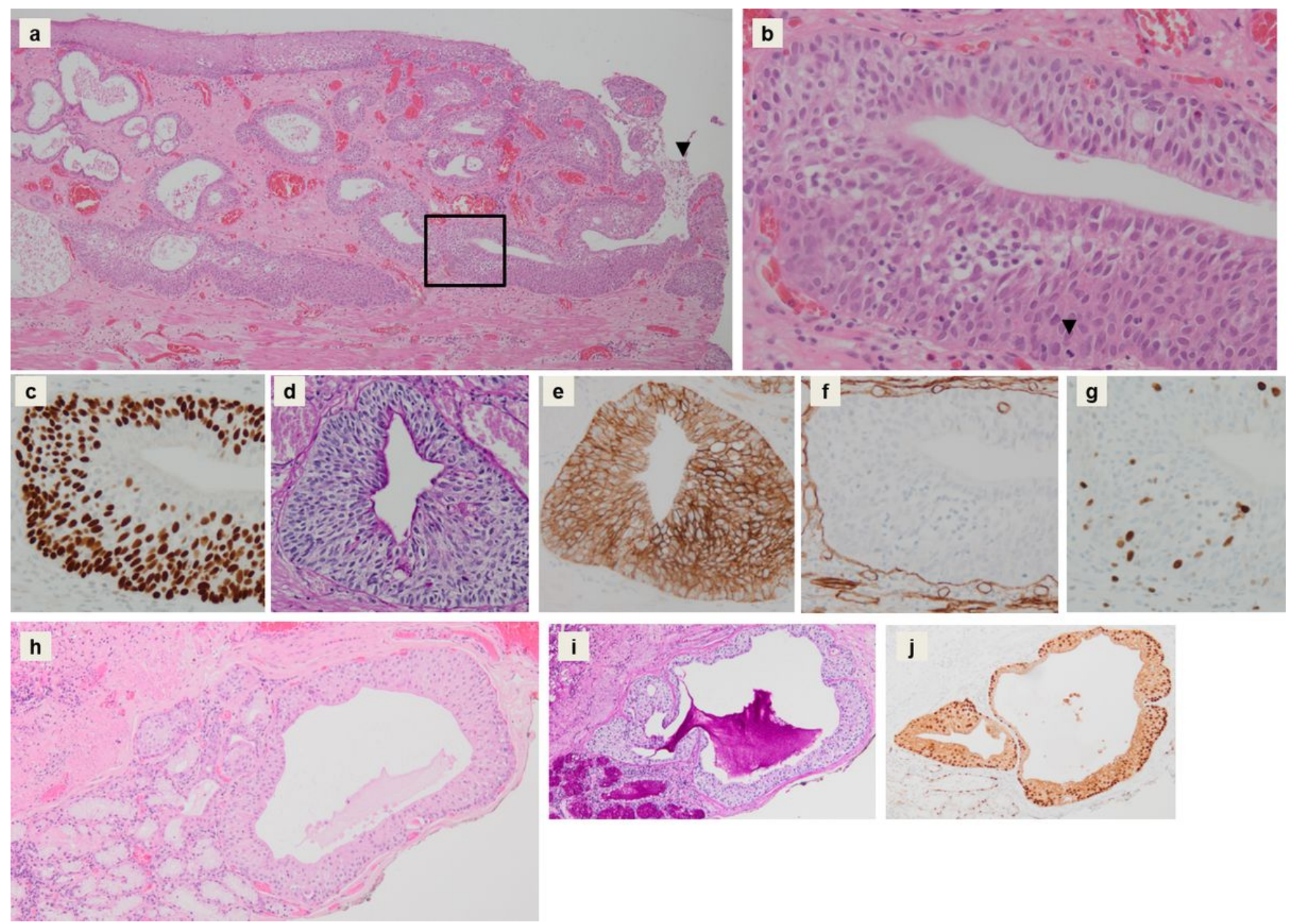

Figure 2

a. Florid ISM with cystic pattern and ISM located in a duct with opening at the epithelial surface (arrow head) (hematoxylin eosin, 40x). b. Inset of Fig. 2a. ISM composed of multilayered immature squamous cells exhibiting slight atypia; a mitosis is observed in the basal layer (arrow head) (hematoxylin eosin, 200x). c. p63 immunostaining positive in immature squamous cells and negative in mucus-secreting cells (200x). d. PAS-diastase staining showing secretion of mucus at the apical part of the epithelium (200x). e. Ber-EP4 immunostaining diffusely positive in ISM (200x). f. Intact and continuous basal membrane demonstrated by Collagen IV immunostaining (200x). g. Ki67 immunostaining positive in a few cells located in the basal and parabasal layers (200x). h. Submucosal glands with stratified duct (100x). i. PAS diastase is positive in epithelium of the glands but not of the ducts (100x). j. p63 immunostaining (100x). 

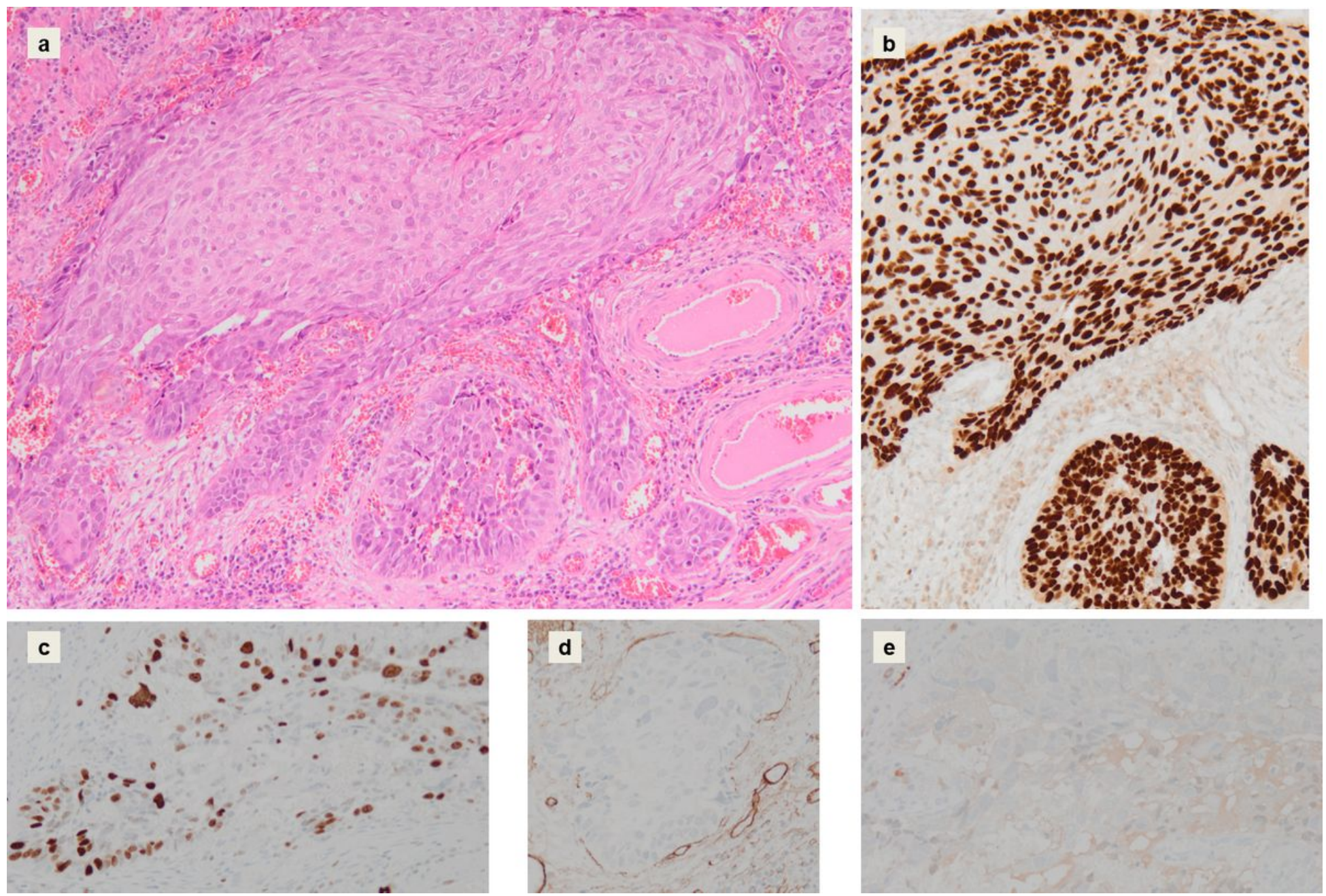

Figure 3

a. Poorly differentiated eSCC organized in solid sheets (100x). b. p63 immunostaining positive in all neoplastic cells (100x). c. Ki-67 immunostaining positive in numerous neoplastic cells (200x). d. Collagen IV immunostaining demonstrating disruption of the basal membrane (200x). e. Ber-EP4 immunostaining negative in the neoplastic cells (200x)

\section{Supplementary Files}

This is a list of supplementary files associated with this preprint. Click to download.

- checklist.pdf 